\title{
Higher Resolution Charge Detection Mass Spectrometry
}

Aaron R. Todd, Lauren F. Barnes, Kim Young, Adam Zlotnick, and Martin F. Jarrold*

Chemistry Department, Indiana University, Bloomington, Indiana 47405 USA

mfj@indiana.edu

Supporting Information 


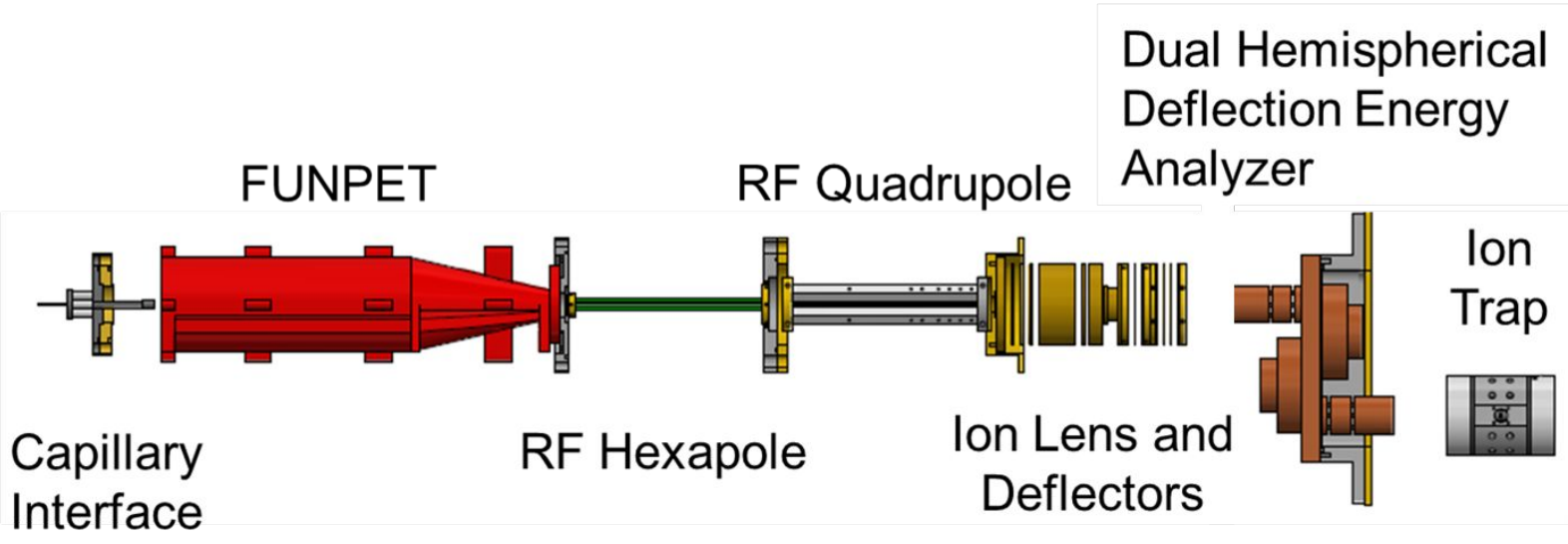

Supplementary Figure S1. A schematic diagram showing the main components of the second generation CDMS instrument used for the high resolving power measurements. 


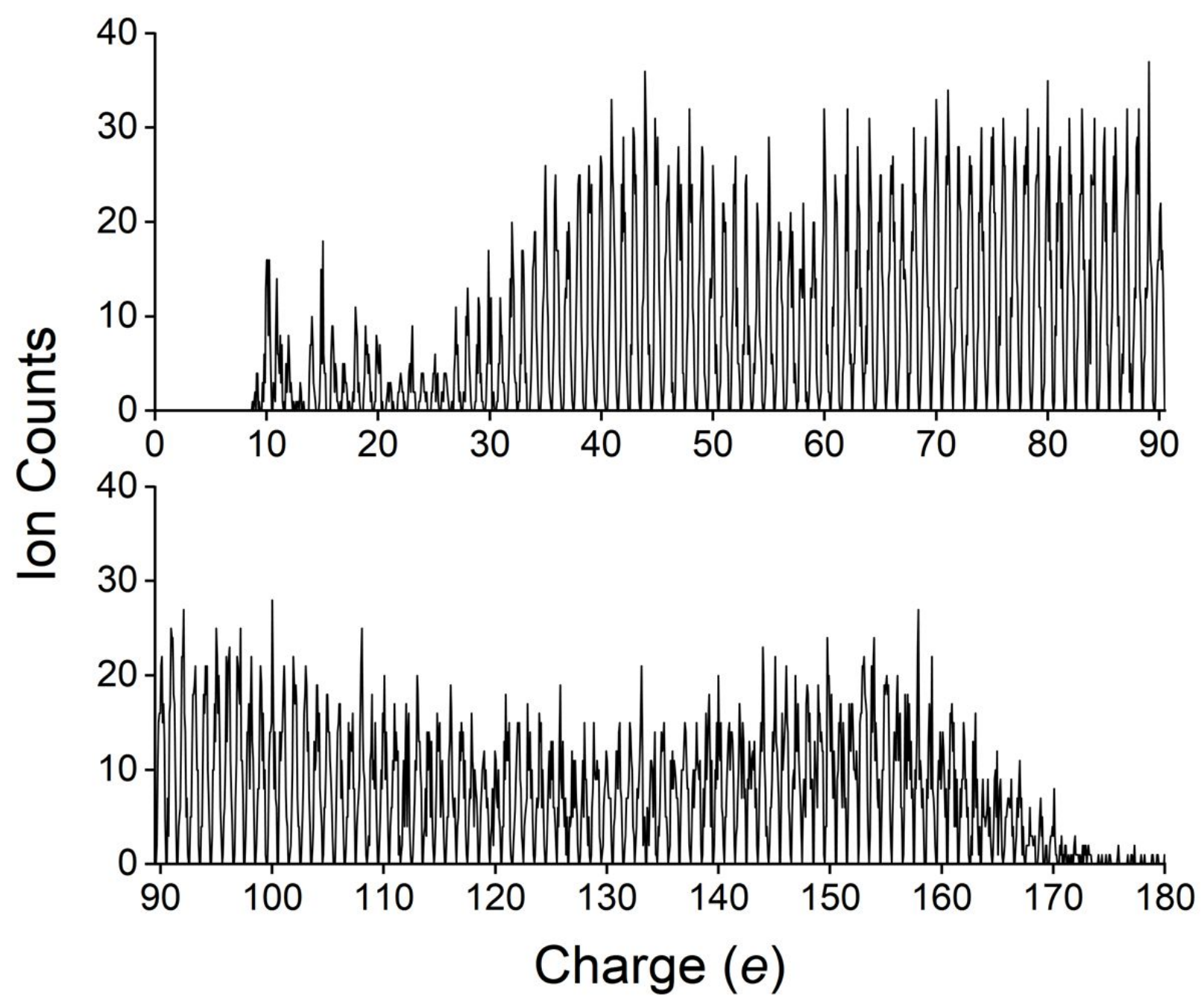

Supplementary Figure S2. Charge spectrum for the mass distribution in Figure 2 of the manuscript. The bin size is 0.075 e. The baseline charge resolution, 0.188 e RMSD, allows for assignment of integer charge values with an error rate of 1 in 120 or $0.83 \%$. 


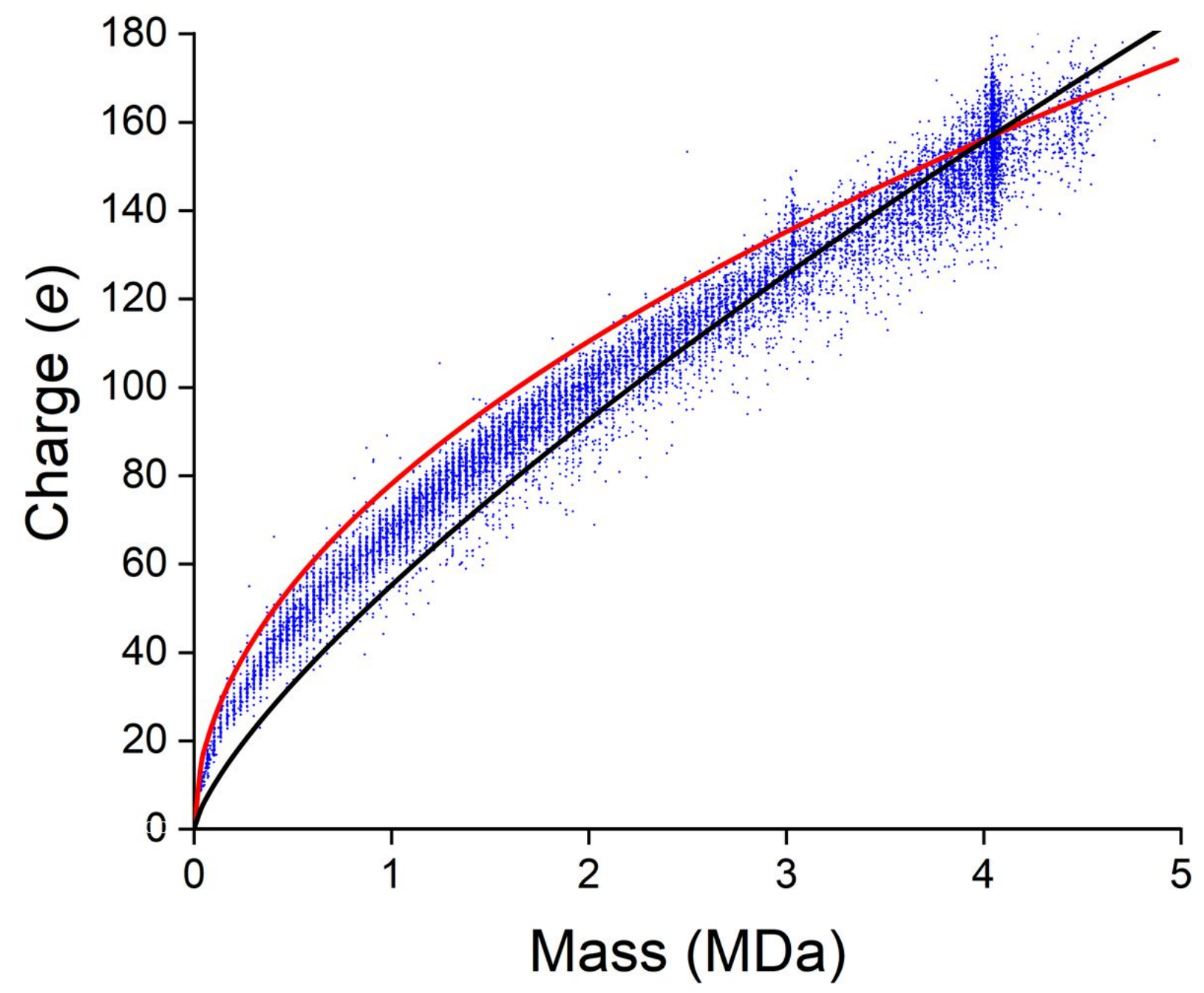

Supplementary Figure S3. Charge versus mass scatter plot for the mass distribution in Figure 2 of the manuscript. Each point is the mass and charge for a single ion. The black and red lines are model calculations using the Rayleigh limit where the maximum charge $\left(z_{R}\right)$ is proportional to $R^{3 / 2}$. For a sphere of uniform density, $z_{R}=C_{s p h} \times m^{1 / 2}$, where $C_{s p h}$ is a constant. The red line shows the charge dependence predicted with $C_{s p h}$ selected to match the average charge of the $T=4$ capsid. However, the capsids are hollow shells rather than solid spheres and the black line shows the dependence predicted for a shell of uniform thickness. In this case, $z_{R}=C_{s h l} \times m^{3 / 4}$, and the constant $C_{s h l}$ was again selected to match the average charge of the $T=4$ capsid. 